\title{
Symposium for Smooth Muscle and Vascular Endothelium
}

\author{
Fumiko SEKIGUCHI ${ }^{1}$ \\ ${ }^{1}$ Division of Physiology and Pathophysiology, School of Pharmaceutical Sciences, Kinki \\ University, 3-4-1 Kowakae, Higashi-Osaka, Osaka 577-8502, Japan
}

In honor of Dr. Satoru SUNANO on his retirement from the Faculty of Pharmaceutical Sciences at Kinki University, a Seminar on Smooth Muscle \& Vascular Endothelium was held in Osaka, Japan on the 10th and 11th of May, 2003. Many outstanding smooth muscle researchers assembled together to share the results of some of their recent research with us all.

Dr. SUNANO has contributed greatly to our knowledge of both smooth muscle and the role played by the vascular endothelium. His most notable works concentrate on the alteration of the properties of vascular and non-vascular tissues in spontaneously hypertensive rats (SHR) and stroke-prone SHR (SHRSP). Analysis of the properties of the vasculature in these pathological animal models has supplied important data for the understanding of the genetic development of hypertension and also of the cellular mechanisms involved in the alteration of vascular tissues during hypertension. One of the highlights of his work has been the finding that $\mathrm{Ca}$ handling properties are altered not only in vascular and non-vascular smooth muscle but also in the sarcoplasmic reticulum of skeletal muscle. These findings indicate that there is functional alteration of the whole body during the development of hypertension, which will help the clinical treatment of patients suffering from high blood pressure.

Dr. SUNANO was born and grew up in Tanabe, Wakayama prefecture. After graduating from the Tanabe high-school, he entered the Sapporo Medical School to become a medical doctor. He received clinical training for a couple of years, and was then promoted to be an assistant in the Department of Physiology, with an opportunity to work on smooth muscle. It should be mentioned that the head of the department at that time was Professor E. Miyazaki who was one of the outstanding pioneers of smooth muscle research in Japan and was the president of the $22^{\text {nd }}$ Annual Meeting of the Japan Society of Smooth Muscle Research. This was at the time when Dr. Hirosi Kuriyama, one of the pioneers of smooth muscle physiology, returned to Japan from the University of Oxford, UK, and introduced the study of smooth muscle electrophysiology to the Physiology Department of the Kyushu University at Fukuoka. Shortly afterwards, Dr. SUNANO visited Fukuoka to learn this new technique for studying smooth muscle physiology. He worked for two years (1971-1973) with Professor H. Liillman at Kiel University, Germany, and then returned to an appointment as Associate Professor of Physiology in the Sapporo Medical School. In 1983, Dr. SUNANO was promoted to become the Professor and Chairman of the Physiology section of the Hypertension Institute of Kinki University. This was when he started working on hypertension using SHR.

Dr. S. Sunano has also made every effort to further smooth muscle research in Japan as an active member of the Japan Society of Smooth Muscle Research. He has worked tirelessly as the Chief Editor of the Journal of Smooth Muscle Research and has attracted articles of high standard to this journal. He also contributed to the Society as the President of the $29^{\text {th }}$ Annual Meeting. It has been a special tribute to Dr. SUNANO that so many exciting papers were presented at this symposium. Dr. SUNANO has been greatly honored to have the symposium at such a high level of excellence. Therefore, we are pleased to be able to publish some of those outstanding papers in the Journal of Smooth Muscle Research. 